\title{
Lethal host-versus-graft disease and hypereosinophilia in the absence of MHC I-T-cell interactions
}

\author{
Jérôme D. Coudert, ${ }^{1}$ Gilles Foucras, ${ }^{1}$ Cécile Demur, ${ }^{2}$ Christiane Coureau, ${ }^{1}$ Catherine \\ Mazerolles, ${ }^{3}$ Georges Delsol, ${ }^{3}$ Philippe Druet, ${ }^{1}$ and Jean-Charles Guéry ${ }^{1}$ \\ ${ }^{1}$ Institut National de la Santé et de la Recherche Médicale (INSERM) U28, Institut Fédératif de Recherche 30, and \\ Université Paul Sabatier, Toulouse, France \\ ${ }^{2}$ Hematology Laboratory, and \\ ${ }^{3}$ Department of Pathology, Centre National de la Recherche Scientifique (CNRS) UPR 2163, Hôpital Purpan, \\ Toulouse, France
}

Address correspondence to: Jean-Charles Guéry, INSERM U28, Hôpital Purpan, Place du Dr Baylac, 31059 Toulouse, France. Phone: 33-561779296; Fax: 33-561779291; E-mail: Jean-Charles.Guery@purpan.inserm.fr.

Received for publication December 22, 1999, and accepted in revised form March 7, 2000.

\begin{abstract}
Neonatal injection of semiallogeneic spleen cells in $\mathrm{BALB} / \mathrm{c}$ mice induces a self-limited state of chimerism that promotes the differentiation of donor-specific CD4 T cells toward the Th2 phenotype. Here we show that injection of spleen cells from $\beta 2$-microglobulin-deficient (BALB/c $\times$ C57BL/6) F1 mice into BALB/c newborns with a disrupted $\beta 2$-microglobulin $(\beta 2 \mathrm{~m})$ gene results in a lethal lymphoproliferative disorder associated with uncontrolled Th2 response, long-term persistence of donor B cells, and sustained blood eosinophilia. Autoimmune manifestations are also enhanced and characterized by a severe autoantibodymediated glomerulonephritis. Histological examination of the spleen shows a hyperplasia of periarteriolar lymphoid sheaths, with accumulation of eosinophils and basophils, and variable degree of fibrosis. Perivascular lymphoid infiltrates with eosinophils are also found in the lung and are correlated with disease severity. Such abnormalities are almost absent using $\beta 2 \mathrm{~m}$-sufficient mice. These data demonstrate that induction of lymphoid chimerism in the absence of MHC class I-T-cell interactions results in a lethal form of host-versus-graft disease that represents a unique model of Th2-dependent chronic inflammatory disease associated with an hypereosinophilic syndrome in mice.
\end{abstract}

J. Clin. Invest. 105:1125-1132 (2000).

\section{Introduction}

Since the original experiments by Billingham, Brent, and Medawar (1), the model of neonatal tolerance to transplantation antigens has been extensively studied to analyze the different parameters promoting allograft acceptance. Although it has been proposed that in mice neonatally injected with semiallogeneic spleen cells, MHC class I-reactive cytotoxic $\mathrm{T}$ cells are tolerized by clonal deletion $(2,3)$, it is clear that alloantigen-specific, IL-4-secreting CD4 T cells are still present in the periphery (4-7) and are probably responsible for the upregulation of MHC class II molecules on both donor and host $\mathrm{B}$ cells (8). In addition, it has been shown that the differentiation of donor-specific CD4 T cells toward the Th2 phenotype is dependent on the early production of IL-4 in vivo $(5,9)$. Furthermore, blocking endogenous IL-4 was found to restore antidonor cytoxic $\mathrm{T}$ lymphocytes (CTL) activity and abrogated the lymphoid chimerism, thereby resulting in graft rejection $(5,10)$. Another important feature of this model is the development of autoimmune manifestations, known as host-versusgraft (HVG) disease, due to the polyclonal T-B-cell interactions that occur in this model between host Th2 and donor B cells, resulting in a dramatic increase of serum IgE level and in the production of autoantibodies, some of which are found deposited in the kidneys $(11,12)$. However, such serological abnormalities are sponta- neously downregulated by 10 weeks of age due to the disappearance of lymphoid chimerism $(11,13)$.

Because IgE production and Th2 priming are critically dependent on the early production of IL-4 (14-16), we have recently analyzed the requirement for NK1.1 T cells in the generation of alloreactive $\mathrm{Th} 2$ responses in this model (17). For this purpose, lymphoid chimerism was induced in both normal and $\beta 2$-microglobulin-deficient $\left(\beta 2 \mathrm{~m}^{\circ}\right) \mathrm{BALB} / \mathrm{c}$ mice. We showed that similar Th2 priming and IL-4-dependent IgE production developed in both $\beta 2 \mathrm{~m}^{\circ}$ or normal BALB $/ \mathrm{c}$ mice injected at birth with semiallogeneic spleen cells from normal $(B A L B / c$ $\times \mathrm{C} 57 \mathrm{BL} / 6) \mathrm{F} 1$ mice $(\mathrm{CB} 6 \mathrm{~F} 1)$, indicating that $\beta 2 \mathrm{~m}$ dependent NK1.1 $1^{+}$host $\mathrm{T}$ cells were not required for the generation of alloreactive Th2 responses in vivo (17). Interestingly, when $\beta 2 \mathrm{~m}^{\circ} \mathrm{CB} 6 \mathrm{~F} 1$ spleen cells were injected into $\mathrm{BALB} / \mathrm{c} \beta 2 \mathrm{~m}^{\circ}$ mice, the IgE response was even exacerbated and associated with an increased splenomegaly (17). Thus, we hypothesized that MHC class I-T-cell interactions could play a crucial role in this model by regulating Th2 immunity and its associated immunological disorders.

To analyze the role of MHC class I-reactive host T cells interacting with donor cells, we have compared 2 combinations, in which wild-type (WT) or $\beta 2 \mathrm{~m}^{\circ}$ newborn $\mathrm{BALB} / \mathrm{c}$ mice were injected either with normal or with $\beta 2 \mathrm{~m}^{\circ} \mathrm{CB} 6 \mathrm{~F} 1$ splenocytes, respectively. Data presented 
here demonstrate that the absence of MHC class I-T-cell interactions leads to spared Th2 immunity that results in accelerated mortality and higher incidence of proteinuria due to antibody-mediated glomerular lesions. The uncontrolled Th2 response of host $\mathrm{CD} 4 \mathrm{~T}$ cells is associated with the maintenance of B-cell lymphoid chimerism and with sustained hypereosinophilia in adult mice. Thus, our data support the hypothesis that CD8 T cells might play a more active role than previously anticipated by regulating donor B-cell persistence and/or Th2-cell development during neonatal induction of lymphoid chimerism in mice, thereby preventing chronic Th2 responses and its associated hypereosinophilic syndrome.

\section{Methods}

Mice, neonatal injection of semiallogeneic spleen cells. BALB/c $\left(\mathrm{H}-2^{\mathrm{d}}\right)$ and $(\mathrm{BALB} / \mathrm{c} \times \mathrm{C} 57 \mathrm{BL} / 6) \mathrm{F} 1(\mathrm{CB} 6 \mathrm{~F} 1)\left(\mathrm{H}-2^{\mathrm{bxd}}\right)$ mice were purchased from Centre d'Elevage R. Janvier (Le Genest St. Isle, France). $\mathrm{H}-2^{\mathrm{b}}$ mice with disrupted $\beta 2 \mathrm{~m}$ genes (18) were back-crossed to $\mathrm{BALB} / \mathrm{c}$ mice as described previously (19). $\beta 2 \mathrm{~m}^{\circ}$ mice on BALB/c background were used after 9 back-crosses. BALB $/ \mathrm{c} \beta 2 \mathrm{~m}^{\circ}$ and $\mathrm{CB} 6 \mathrm{~F} 1 \beta 2 \mathrm{~m}^{\circ}$ mice were bred and maintained in our own animal facility. $\mathrm{BALB} / \mathrm{c}$ and $\mathrm{BALB} / \mathrm{c} \beta 2 \mathrm{~m}^{\circ}$ were injected intraperitoneally within the first 24 hours of life, with $50 \times 10^{6}$ spleen cells from 8- to 12 -week-old normal or $\beta 2 \mathrm{~m}^{\circ} \mathrm{CB} 6 \mathrm{~F} 1 \mathrm{mice}$, respectively. Control animals were left untreated.

Quantitation of serum IgE. Serum IgE concentration was quantified by a sandwich ELISA as described previously (17). Serum IgE concentrations measured either in WT or in $\beta 2 \mathrm{~m}^{\circ}$ control mice were less than $100 \mathrm{pg} / \mathrm{mL}$. We considered that the IgE response was upregulated when $\operatorname{IgE}$ concentration was greater than $1 \mu \mathrm{g} / \mathrm{mL}$.

Albuminuria determination. Urine was collected at 10-16 weeks of age, and the presence of mouse serum albumin (MSA) was determined by competitive ELISA. Briefly, polyvinyl microtiter plates were coated with 2 $\mu \mathrm{g} / \mathrm{mL}$ mouse serum albumin (Sigma Chemical Co., St. Louis, Missouri, USA). Urines were first incubated with an appropriate concentration of rabbit anti-mouse albumin antibody (Cappel Laboratories, Cochranville, Pennsylvania, USA), then transferred onto MSA-coated plates. After incubation, bound rabbit anti-MSA IgG were stained with a peroxidase-conjugated sheep antirabbit IgG (Serotec, Oxford, United Kingdom), and revealed by 3,3'-5, 5'-tetramethylbenzidine (Fluka Chemical Co., Buchs, Switzerland). Albumin concentration was determined using MSA (Sigma Chemical Co.) to generate a standard curve. The concentration of albumin in WT or $\beta 2 \mathrm{~m}^{\circ}$ control mice was routinely less than $5 \mu \mathrm{g} / \mathrm{mL}$. Mice were considered albuminuric when albumin concentration was greater than $15 \mu \mathrm{g} / \mathrm{mL}$.

Indirect immunofluorescence analysis. Kidney cryostatsections $(4 \mu \mathrm{m})$ were performed with a Cryocut 1800 apparatus (Reichert-Jung, Cambridge Instruments GmbH, Nussloch, Germany) after OCT compound (BDH Laboratories, Poole, United Kingdom) embedding. Glomerular IgM and IgG deposits were assessed by indirect immunofluorescence on cryostat sections stained with FITC-labeled rat anti-mouse kappa light chain OX-20 mAb (20), FITC-labeled mouse IgG subclasses-specific (IgG1, IgG2a, IgG2b) antibody (Southern Biotechnology Associates, Birmingham, Alabama, USA), or FITC-labeled rabbit anti-mouse IgM antibody (Meloy Laboratories, Springfield, Virginia, USA).

Histological and cytological analysis. All major organs (liver, heart, intestine, kidney, spleen, and lung) were fixed in 10\% formalin and paraffin sections were stained with hematoxylin and eosin (H\&E), periodic acid-Schiff (PAS), and Giemsa. Histological analysis was performed by 2 pathologists who did not have the knowledge of the different experimental groups. Blood white cell counts and spleen cell counts were performed with an automated counter (STKS Coulter, Beckman Coulter, Roissy, France). Differential cell determinations were carried out using spleen cell suspensions that were cytocentrifugated on a Cytospin 3 Shandon apparatus (Life Sciences International, Runcom, United Kingdom), or standard blood smears fixed with methanol and stained with Giemsa. Interpretation was done by 1 of us blinded to the different experimental groups.

Flow cytometric analysis. After red blood cell lysis, spleen cells were incubated with optimal concentrations of FITC-, PE- or biotin-conjugated $\mathrm{mAb}$ for 30 minutes at $4{ }^{\circ} \mathrm{C}$ in PBS containing 5\% FCS, $0.1 \%$ sodium azide. The following mAb's were used: FITC-GK1.5 anti-CD4 (TIB 207; American Type Culture Collection [ATCC], Rockville, Maryland, USA), PE-Ly2 anti-CD8 $\alpha$ (PharMingen, San Diego, California, USA), PE-RA36B2 anti-B220 (PharMingen), FITC-AF6-120.1 anti-I$\mathrm{A}^{\mathrm{b}}$ (PharMingen), FITC-14.4.4S anti-I-E (ATCC, HB 32), PE-Ly24 (Pgp1) anti-CD44 (PharMingen), and biotin anti-Ly22 (MEL-14) anti-CD62L (PharMingen). Cells were then washed and stained with streptavidinCyChrome (PharMingen). Data were collected on 10,000 living cells as determined by forward and side scatter intensity on a XL Coulter cytometer (Beckman Coulter) and analyzed using the CellQuest software (Becton Dickinson, Mountain View, California, USA).

CD4 T-cell enrichment. Spleen cells were stained with an appropriate concentration of KT1.5 (21), anti-B220 RA33A1 (TIB 146; ATCC), anti-MHC class II M5/114 (TIB 120; ATCC), and anti-Mac-1 M1/70 (TIB 128; ATCC) $\mathrm{mAb}$, washed, and incubated with sheep anti-rat IgG M450 Dynabeads (Dynal, Oslo, Norway). CD4 T cells were negatively selected by magnetic cell separation.

In vitro CD4 T-cell stimulation and cytokine assays. For cytokine production analysis, 96-well culture plates (Costar, Cambridge, Massachusetts, USA) were coated with 145-2C11 anti-CD3e (CRL-1975; ATCC) at a concentration of $1 \mu \mathrm{g} / \mathrm{mL}$. CD4-enriched splenic T cells from neonatally injected and control mice were stimulated at $2 \times 10^{5}$ cells per well. Culture medium was HL-1 synthetic medium (Hycor, Irvine, California, USA) supplemented with $2 \mathrm{mM}$ L-glutamine and $50 \mu \mathrm{g} / \mathrm{mL}$ gentamicin (Sigma Chemical Co.). Cultures were incubated for 2 days in a humidified atmosphere of $5 \% \mathrm{CO}_{2}$ in air. 
Supernatants from replicate cultures, usually 3-4 wells, were collected after 48 hours and pooled for cytokine analysis. IFN- $\gamma$, IL-4, IL-5, and IL-10 were quantified by 2 -site sandwich ELISA as described previously (17). Cytokines were quantified from 2-3 titration points using standard curves generated with purified recombinant mouse cytokines, and results were expressed as cytokine concentration in nanograms or picograms per milliliter. Detection limits were $15 \mathrm{pg} / \mathrm{mL}$ for IFN- $\gamma$ and IL-4, $3 \mathrm{pg} / \mathrm{mL}$ for IL-5, and $30 \mathrm{pg} / \mathrm{mL}$ for IL-10.

Competitive RT-PCR. Total RNA was extracted using TRIzol Reagent (GIBCO-BRL, Cergy-Pontoise, France) according to manufacturer's recommendations. RT was performed using Moloney Murine Leukemia Virus RT (GIBCO-BRL) and oligo-dT primers. Semiquantitative PCR determination for hypoxanthine-guanine phosphoribosyl transferase (HPRT) and IL-4 genes was performed as described elsewhere (22). Data were expressed as the ratio of IL-4 over HPRT mRNA levels. Statistical analysis. Statistically significant differences between groups of continuous variables were analyzed using the Mann-Whitney $U$ test. Categorical variables and survival curves were compared, respectively, by the Fisher's exact test and by the log-rank test.

\section{Results}

Effects of $\beta 2 m$ deficiency in host versus graft disease. Injection of semiallogeneic spleen cells in newborn BALB/c mice stimulates host-derived alloreactive $\mathrm{Th} 2$ cells that react with MHC class II alloantigens on donor B cells. This cognate interaction induces a polyclonal B-cell activation characterized by increased levels of serum IgE and autoantibody production $(11,12)$. As shown in Figure 1, no difference in serum levels could be observed at 4 weeks of age between the WT (Figure 1a) and the $\beta 2 \mathrm{~m}^{\circ}$ (Figure 1b) combinations. In striking contrast, whereas serum IgE levels were spontaneously downregulated by 8 weeks of age in the CB6F1-injected WT mice (Figure 1a), induction of lymphoid chimerism in the complete absence of $\beta 2 \mathrm{~m}$ dependent $\mathrm{T}$ cells resulted in uncontrolled IgE responses that remained high until 12 weeks of age (Figure $1 \mathrm{~b}$ ). The incidence of $\operatorname{IgE}$ response, at 4 weeks of age, in the $\mathrm{CB} 6 \mathrm{~F} 1 \rightarrow \mathrm{BALB} / \mathrm{c}$ and $\mathrm{CB} 6 \mathrm{~F} 1 \beta 2 \mathrm{~m}^{\circ} \rightarrow \mathrm{BALB} / \mathrm{c} \beta 2 \mathrm{~m}^{\circ}$ combinations were $65 \%(37 / 57)$ and $83 \%(44 / 53)$, respectively. Interestingly, whereas most $\mathrm{BALB} / \mathrm{c}$ mice injected at birth with CB6F1 spleen cells remained healthy and survive, more than $50 \%$ of mice died by 15 weeks of age in the $\beta 2 \mathrm{~m}^{\circ}$ combination (Figure 1c). This increased mortality was associated with an higher incidence of albuminuria (Figure 1d). Urine albumin levels were above $100 \mu \mathrm{g} / \mathrm{mL}$ in $66 \%$ of albuminuric mice, with a maximum of 16 $\mathrm{mg} / \mathrm{mL}$ in 1 mouse (data not shown). Immunofluorescence analysis on kidney cryostat sections demonstrated the presence of heavier glomerular Ig deposits in neonatally injected $\beta 2 \mathrm{~m}^{\circ}$ compared with WT mice (Figure 2, b and d), that were mainly composed of IgG1 and IgM (data not shown). Moreover, whereas in the WT combination kidney bound Ig were mostly found in the mesangium (Figure 2b), both mesangial and subendothelial granular
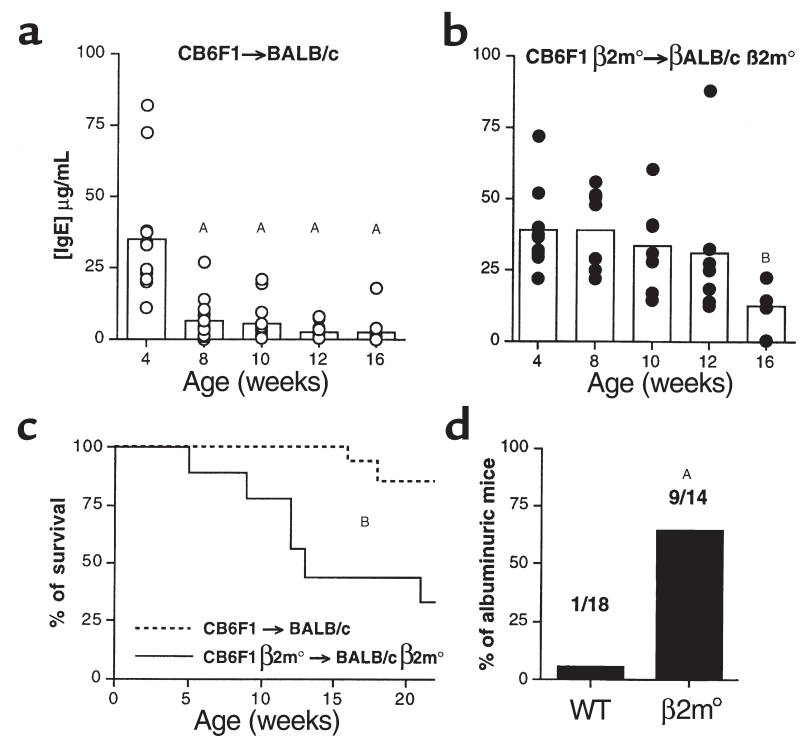

d

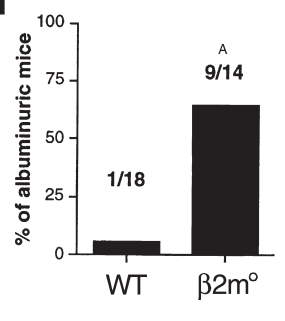

\section{Figure 1}

Persistence of IgE production, high mortality rate, and increased incidence of albuminuria in $\beta 2 \mathrm{~m}^{\circ} \mathrm{BALB} / \mathrm{c}$ mice injected at birth with $\beta 2 m^{\circ}$ CB6F1 splenocytes. Normal (a) and $\beta 2 m^{\circ}$ (b) BALB/c mice were injected at birth with $50 \times 10^{6} \mathrm{CB} 6 \mathrm{~F} 1$ spleen cells from normal or $\beta 2 \mathrm{~m}^{\circ}$ donors, respectively. Mice were bled at 4-16 weeks of age and IgE were quantified in individual mice serum by ELISA. Statistically significant decrease of IgE concentration was compared between the 8-, 10-, 12-, and 16-week values and the 4-week values. Bars represent means of individual mice. (c) Mortality of neonatally injected $\beta 2 \mathrm{~m}^{\circ}$ (solid line; $n=9$ ) and WT mice (dotted line; $n=13$ ) was followed up until the age of 22 weeks. (d) Albumin was quantified in urine by ELISA at 10-16 weeks of age. Mice were considered albuminuric when albumin titer was greater than $15 \mu \mathrm{g} / \mathrm{mL}$. Statistical analyses were performed as indicated in Methods. ${ }^{A} P<0.001$; ${ }^{B} P<0.01$

Ig deposits were observed in 7 of the 11 neonatally injected $\beta 2 \mathrm{~m}^{\circ}$ mice examined (Figure $2 \mathrm{~d}$ ), the 4 other mice displayed only mesangial Ig deposits. Unlike BALB/c mice injected with CB6F1 splenocytes, that exhibited only mild mesangial deposits without any cell proliferation or interstitial changes (Figure 2a), glomeruli from 4 of 6 neonatally injected $\beta 2 \mathrm{~m}^{\circ}$ mice examined by light microscopy exhibited heavy mesangial and parietal, mainly subendothelial, deposits (Figure 2c). There was also a double contour appearance after silver impregnation (data not shown). In addition, perivascular infiltrates of mononuclear cells were observed in 2 of the $6 \beta 2 \mathrm{~m}^{\circ}$ mice (data not shown). Renal function appeared normal in these mice, as urea and creatinine blood levels were unchanged (data not shown). Another striking observation of the $\beta 2 \mathrm{~m}^{\circ}$ chimeric model was that these mice developed a much larger splenomegaly compared with the WT combination, which still persisted until 10 weeks of age, with variable degrees of fibrosis (not shown). The increased spleen size in neonatally induced $\beta 2 \mathrm{~m}^{\circ}$ chimeras was associated with a significant augmentation in CD4 $\mathrm{T}$ and $\mathrm{B}$ cell numbers (data not shown).

Spared Th2 immunity is associated with the maintenance of lymphoid chimerism in $\beta 2 m$-deficient mice. Because splenomegaly in this model is likely to be associated with 


\section{Figure 2}

Histological changes and immunofluorescence analysis of kidney. ( $\mathbf{a}$ and $\mathbf{b}$ ) Representative changes in 6 week-old BALB/c mice injected at birth with CB6F1 splenocytes. Moderate mesangial deposits are seen on H\&E staining $(\mathbf{a}, \times 800)$ and after immunostaining for mouse Ig (b, $\times 500)$. (c and d) Representative changes in 6-week-old $\beta 2 \mathrm{~m}^{\circ} \mathrm{BALB} / \mathrm{c}$ mice neonatally injected with $\beta 2 \mathrm{~m}$-deficient CB6F1 spleen cells. Strong mesangial and subendothelial immune complex deposits are seen on PAS staining $(\mathbf{c}, \times 800)$ and after immunostaining for mouse $\lg (\mathbf{d}, \times 500)$.
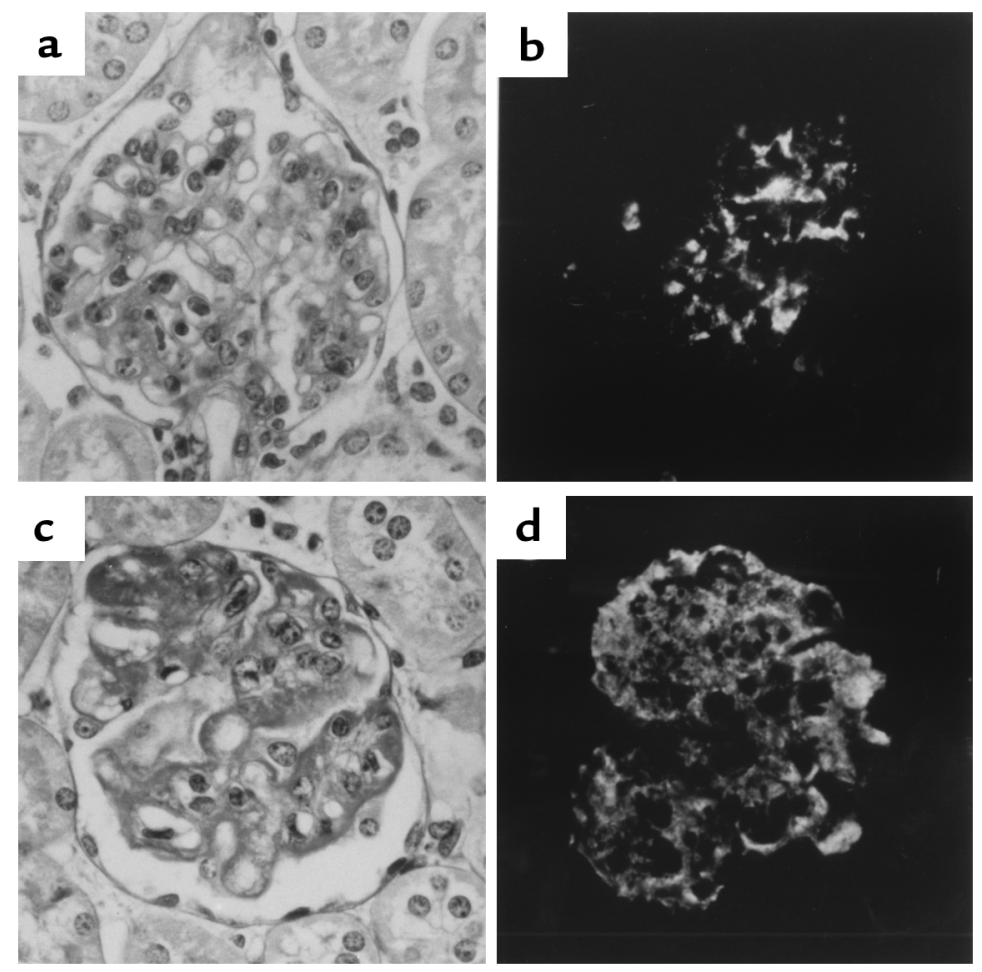

uncontrolled Th2 immunity, we next compared the expression of memory/activation markers on $\mathrm{T}$ and $\mathrm{B}$ cells between these 2 combinations. Results in Figure 3 show that there was a significant increase in antigen-experienced splenic CD 4 T cells in both WT and $\beta 2 \mathrm{~m}^{\circ}$ mice at 6 weeks of age after neonatal induction of lymphoid chimerism. $\mathrm{CD} 44^{\text {high }} \mathrm{CD} 62 \mathrm{~L}^{\text {low }} \mathrm{CD} 4 \mathrm{~T}$-cell numbers were higher in $\mathrm{CB} 6 \mathrm{~F} 1 \beta 2 \mathrm{~m}^{\circ}$-injected $\mathrm{BALB} / \mathrm{c} \beta 2 \mathrm{~m}^{\circ}$ mice compared with the WT combination. This difference was more evident at 10 weeks of age, where number of memory/effector CD4 $\mathrm{T}$ cells were significantly increased in neonatally injected $\beta 2 \mathrm{~m}^{\circ}$ but not WT mice. Analysis of the splenic B-cell compartment showed that the expression of I-E molecules on $B$ cells was significantly higher in neonatally injected $\beta 2 \mathrm{~m}^{\circ}$ compared with WT mice at both 6 and 10 weeks of age (Figure 4a). Because MHC class II expression on B cells is upregulated by IL-4 $(23,24)$, we looked for the presence of IL-4 transcripts in splenic CD4 T cells. Data in Figure 4b demonstrate that IL-4 mRNA were readily detectable in the majority $(85 \%)$ of 6 week-old neonatally induced $\beta 2 \mathrm{~m}^{\circ}$ chimeras, compared with $22 \%$ in the WT combination. IL-4 is likely to be produced by allospecific Th 2 cells interacting with donor $\mathrm{F} 1$ $\mathrm{B}$ cells, resulting in donor B-cell differentiation to Ig-producing cells. Indeed, the number of plasmocytes was selectively increased in the spleen of 6-week-old neonatally injected $\beta 2 \mathrm{~m}^{\circ}$ mice (Figure $4 \mathrm{c}$ ) and was correlated with the persistence of significantly higher levels of B-cell chimerism (Figure 4d). To analyze further the effect of the absence of MHC class I-T-cell interactions in the development of Th2 cells in this model, we measured type- 2 cytokine production by splenic CD4 T cells from 6-weekold mice after stimulation with plate bound anti-CD3e $\mathrm{mAb}$. Data in Figure 5 show that there was a 4-fold upreg-

\section{Figure 3}

Accumulation of CD44hi/CD62 low $T$ lymphocytes in neonatally injected $\beta 2 \mathrm{~m}^{\circ} \mathrm{BALB} / \mathrm{c}$ mice. WT and $\beta 2 \mathrm{~m}^{\circ} \mathrm{BALB} / \mathrm{c}$ mice were injected at birth with normal or $\beta 2 \mathrm{~m}$-deficient $\mathrm{CB} 6 \mathrm{~F} 1$ spleen cells as in Figure 1. Uninjected WT and $\beta 2 \mathrm{~m}^{\circ} \mathrm{BALB} / \mathrm{c}$ mice were used as control. Splenocytes from 6 - to 10 -week-old neonatally injected and control mice were analyzed by flow cytometry to quantify the CD44hi/CD62L low memory/effector $C D 4^{+} \mathrm{T}$ cells. Bars represent means \pm SEM of individual mice (4-12 mice per group). Numbers in brackets indicate the fold increase of neonatally induced chimera versus control mean values. ${ }^{A} P<0.05 ;{ }^{B} P<0.01$.
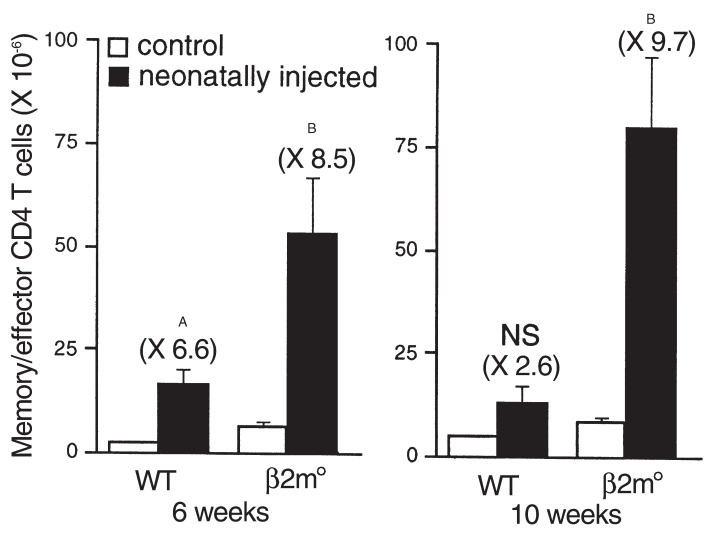


\section{Figure 4}

Upregulation of MHC class II on B cells, increased number of plasmocytes, and IL4-mRNA are associated with the maintenance of lymphoid chimerism in $\beta 2 \mathrm{~m}^{\circ} \mathrm{BALB} / \mathrm{c}$ mice. (a) I-E expression level on splenic B lymphocytes from 6- and 10-week-old neonatally injected and control mice was evaluated by flow cytometry. Data are expressed as fold increase versus control (mean \pm SEM) of 4-12 mice per group. (b) IL4-mRNA expression in negatively enriched CD4 splenic T cells, from 6-week-old neonatally injected WT and $\beta 2 \mathrm{~m}^{\circ}(n=14)$ mice, was quantified by semiquantitative RT-PCR. Data are expressed as the ratio of IL-4/HPRT mRNA levels from 9-14 individual mice per group. (c) Cytospins were prepared from spleen cells suspensions from 6-week-old mice; total plasmocyte numbers were calculated from 4-7 individual mice per group. (d) Percentages of $\mathrm{I}_{-\mathrm{A}^{\mathrm{b}+}}$ splenic B220 $0^{+}$cells from 6-week-old WT $(n=$ $16)$ and $\beta 2 \mathrm{~m}^{\circ}(n=9)$ mice injected at birth with semiallogeneic splenocytes were evaluated by flow cytometry analysis. Bars represent means of individual mice. ulation of IL-4 production in F1-injected WT mice compared with uninjected control animals. In striking contrast, IL-4 secretion was dramatically increased (54-fold) in the neonatally induced $\beta 2 \mathrm{~m}^{\circ}$ chimeras. The lower IL-4 production in control $\beta 2 \mathrm{~m}^{\circ}$ compared with WT BALB/c mice may be explained by the absence of NK T cells that promptly produce this cytokine in response to TCR ligation (25). As for IL-4, IL-10 secretion upon TCR engagement was strongly increased in CD4 T cells from both combinations, but this was more evident in $\beta 2 \mathrm{~m}^{\circ}$ mice. On the other hand, the amounts of IL- 5 produced by CD 4 $\mathrm{T}$ cells was significantly upregulated only in $\beta 2 \mathrm{~m}^{\circ}$ chimeras (Figure 5). IFN- $\gamma$ synthesis was slightly increased (2- to 3-fold) in neonatally injected compared with control mice both in the WT and $\beta 2 \mathrm{~m}^{\circ}$ combinations (data not shown). Thus, the induction of lymphoid chimerism in the absence of MHC class I-T-cell interactions led to the accumulation in the spleen of CD4 T cells with a memory phenotype probably as a consequence of the long-term persistence of donor $\mathrm{B}$ cells, resulting in chronic alloantigenic stimulation and increased type- 2 cytokine production by CD $4 \mathrm{~T}$ cells.

Hypereosinophilic syndrome develops after induction of lymphoid chimerism in the absence of MHC class I-T-cell interactions. Because the uncontrolled Th2 response that devel-
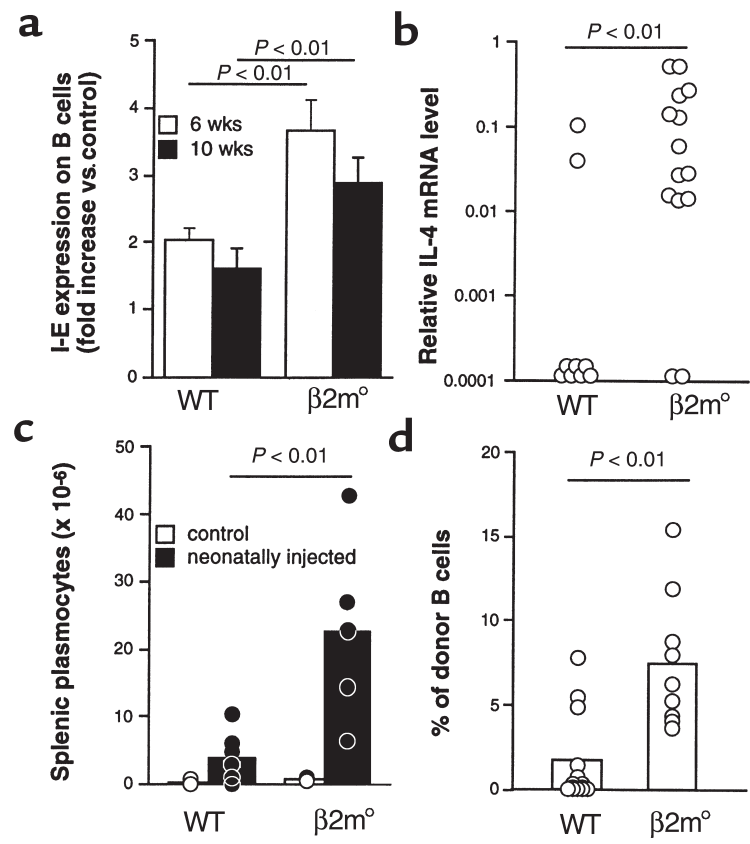

ops in $\beta 2 \mathrm{~m}^{\circ}$ chimeric mice is associated with an increased splenomegaly with signs of fibrosis in most animals, a morphological analysis was performed. Data in Figure 6 show that although neonatally injected WT mice exhibited minimal change in spleen morphology (Figure 6a), a considerable modification of spleen architecture was observed in chimeric $\beta 2 \mathrm{~m}^{\circ} \mathrm{BALB} / \mathrm{c}$ mice (Figure $6 \mathrm{~b}$ ). Such changes were characterized by a strong hyperplasia of periarteriolar lymphoid sheaths (PALS) (Figure 6b) and by prominent eosinophil- (Figure 6c), basophil-, and/or mastocyte-containing (Figure 6d) infiltrates in the majority $(>80 \%)$ of $\beta 2 \mathrm{~m}^{\circ}$ chimeras. These modifications were associated with variable degree of fibrosis (Figure $6 \mathrm{~b}$ and data not shown). Massive perivascular lymphoid infiltration (Figure 6f) and tissue eosinophilia (data not shown) were also occasionally found in the lung ( 2 of 5 $\beta 2 \mathrm{~m}^{\circ}$ chimeric mice tested) and were correlated with disease severity (data not shown). Histological analysis of others organs such as liver, heart, and intestine did not reveal significant abnormalities (data not shown). Cytological analysis confirmed the increased eosinophilia in the spleen of neonatally induced $\beta 2 \mathrm{~m}^{\circ}$ chimeras compared with F1-injected WT mice (Figure 7a). Furthermore, a significant increase in eosinophil number was detected in the blood of 6 - to 10 -week-old $\beta 2 \mathrm{~m}^{\circ}$ chimeric

\section{Figure 5}

Th2-type cytokines production by in vitro stimulated CD4 T lymphocytes. Negatively enriched splenic CD4 T cells from 6-week-old control and neonatally injected mice were stimulated with plate-bound antiCD3 $\varepsilon$ mAb for 48 hours. IL-4, IL-10, and IL- 5 were quantified in culture supernatants by ELISA. Results are expressed as mean \pm SEM cytokine concentration $(\mathrm{ng} / \mathrm{mL})$ from 4-7 mice per group. Numbers in brackets indicate the fold increase of neonatally injected versus control mean values. ${ }^{A} P<0.05 ;{ }^{B} P<0.01$. 
mice (Figure 7b). Such abnormalities were barely detectable or absent in the majority of F1-injected $\beta 2 \mathrm{~m}$ sufficient mice. Thus, the uncontrolled type- 2 immune response that develops in $\beta 2 \mathrm{~m}^{\circ} \mathrm{BALB} / \mathrm{c}$ mice injected at birth with semiallogeneic $\beta 2 \mathrm{~m}^{\circ}$ splenocytes leads to an hypereosinophilic syndrome.

\section{Discussion}

The present report demonstrates that induction of lymphoid chimerism in BALB/c mice in the absence of MHC class I-T-cell interactions results in a lethal syndrome associated with uncontrolled Th2 immunity and sustained hypereosinophilia. In addition, these mice develop a severe form of antibody-mediated glomerulonephritis probably as a consequence of autoreactive F1 donor B-cell activation by host allohelper T cells. However, the autoimmune glomerulonephritis is not, or is not only, responsible for the mortality because bio- logic signs of renal failure were absent, and some mice died without proteinuria. We postulate that lung damages may contribute to the increased mortality in $\beta 2 \mathrm{~m}^{\circ}$ chimeric mice. This model markedly contrasts with the mild and self-limited form of HVG disease that occurs in WT BALB/c mice injected with CB6F1 spleen cells $(11,13)$ and represents the first example of a Th2dependent lethal chronic inflammatory disease in mice associated with an hypereosinophilic syndrome.

The mechanisms by which $\beta 2 \mathrm{~m}$ deficiency leads to an uncontrolled Th2 response are likely to be related to the lack of interactions between donor MHC class I molecules and host CD8 T cells. In previous experiments, we have shown that the injection of CB6F1 spleen cells into normal or $\beta 2 \mathrm{~m}^{\circ} \mathrm{BALB} / \mathrm{c}$ mice induces identical Th2type responses (17). This could be explained by the fact that in $\beta 2 \mathrm{~m}^{\circ}$ mice there is a residual population of CD8 $T$ cells that can readily expand after in vivo priming with

\section{Figure 6}

Histological changes in the spleen (a-d) and lung (e and $\mathbf{f}$ ) of 6 -week-old WT ( $\mathbf{a}$ and $\mathbf{e}$ ) and $\beta 2 \mathrm{~m}^{\circ} \mathrm{BALB} / \mathrm{c}$ mice $(\mathbf{b}, \mathbf{c}, \mathbf{d}$, and $\mathbf{f})$ injected at birth with normal or $\beta 2 \mathrm{~m}$-deficient CB6F1 splenocytes, respectively. In comparison with the spleen of WT mice (a, $\times 125)$, a striking hyperplasia of PALS is observed on H\&E staining of $\beta 2 m^{\circ}$ mice $(\mathbf{b}, \times 100)$. On higher magnification $(\times 800)$, one sees large numbers of eosinophils (arrows) (c) in a background of activated lymphoid cells, some showing immunoblastic features (arrowheads). Giemsa staining shows that cells with large basophilic granules (arrows) are also present in PALS (d). Note also the presence of scattered plasma cells (arrowheads). (e and $\mathbf{f}$ ) H\&E staining shows perivascular infiltrates (arrows) consisting of activated lymphoid cells associated with eosinophils and basophils in the lung of a $\beta 2 \mathrm{~m}^{\circ}$ mouse $(\mathbf{f}, \times 100)$. No significant changes are seen in the lung of a WT mouse $(\mathbf{e}, \times 100)$.
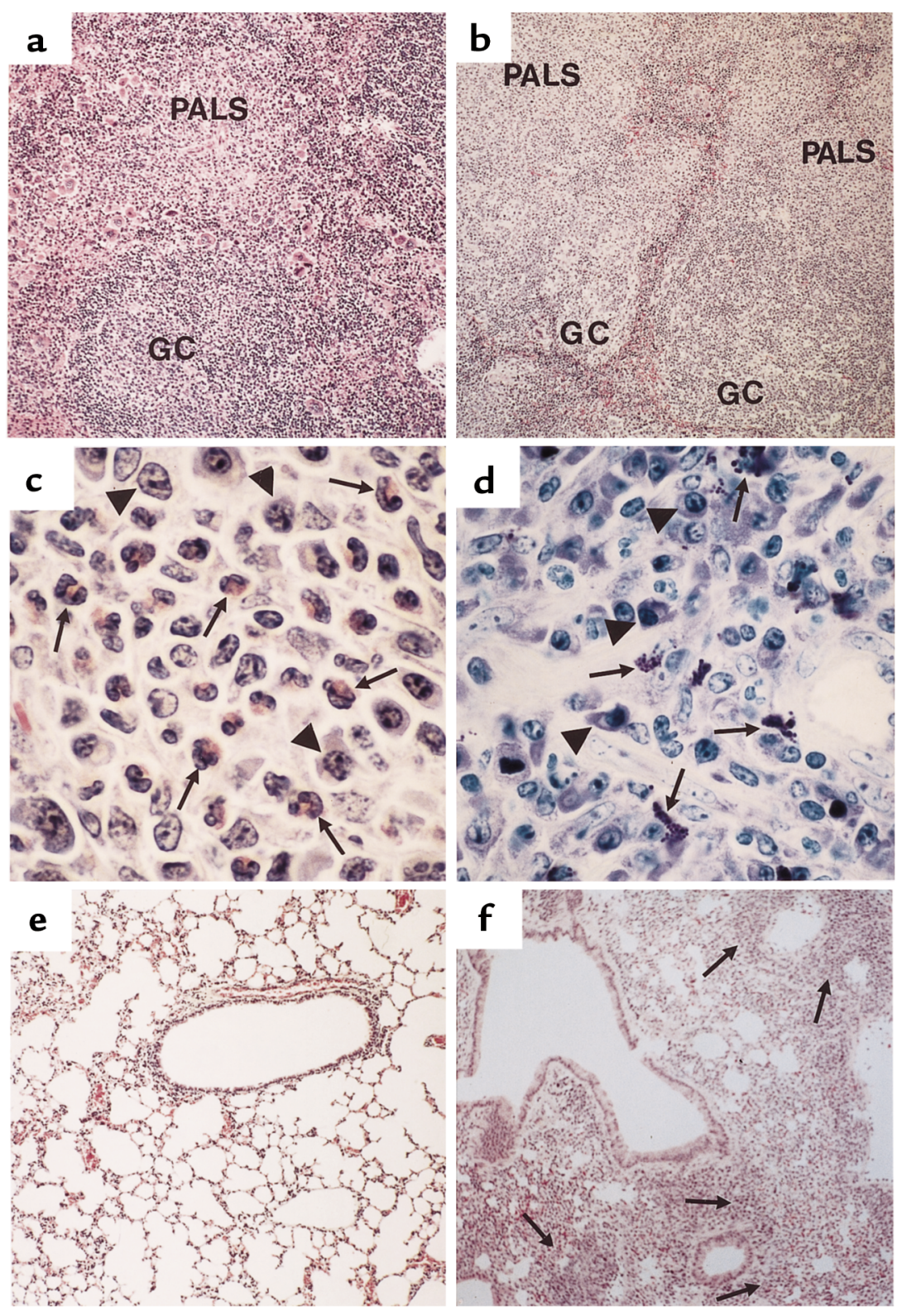
cells expressing either self- or allogeneic-MHC class I molecules $(26,27)$. Thus, to avoid the interference of this minor population of CD8 T cells, MHC class I molecules must also be absent on donor-derived CB6F1 cells. The demonstration that MHC class I-Tcell interactions play a crucial role in the control of Th2 immunity and its associated lethal syndrome is rather unexpected. Indeed, it is widely accepted that after neonatal transplantation, tolerance alloantigenspecific CTLs are deleted in the thymus and in the periphery $(2,3)$. However, the failure to detect allospecific CTL activity in neonatally primed mice could also be explained by a difference in phenotype acquisition in the CD8 compartment rather than clonal deletion. Indeed, it has been shown that under the influence of IL-4, CD8 T cells can develop into noncytotoxic, type-2 cytokines producing cells (28). Alternatively, it may be possible that functional alloreactive CTL cells still persist in tolerized mice, but that their frequency is largely underestimated in limiting dilution analysis. The hypothesis that CD8 T cells might play a crucial role in this model is in apparent contradiction to previously published experiments showing that administration of anti-CD8 $\mathrm{mAb}$ in vivo had no effect on disease severity (29). This could easily be explained by the fact that the few CD $8 \mathrm{~T}$ cells that might have escaped in vivo depletion with anti-CD8 $\mathrm{mAb}$ could still mediate efficient regulatory effector functions. Indeed, as already discussed here, the minor CD8 T-cell population that persist in $\beta 2 \mathrm{~m}^{\circ}$ mice seems to be as effective as conventional CD8 T cells in the control of Th2 responsiveness (17). Taken together, our data support the conclusion that MHC class I expression on donor spleen cells and the presence of potentially MHC class I-reactive $\mathrm{T}$ cells in the host are the critical factors that control HVG disease severity induced by neonatal injection of semiallogeneic spleen cells.

Several mechanisms could be proposed to explain how MHC class I-reactive T cells could regulate Th2 immunity in this lethal HVG disease. First, as these abnormalities are strongly correlated with the persistence of B-cell chimerism, it is reasonable to speculate that the main effector function of class I-reactive $\mathrm{T}$ cells would be to eliminate donor-derived antigen-presenting cells (APC), thereby preventing chronic allohelper T-cell activation, resulting in a mild and selflimited disease. Second, the absence of CD8 T-cell activation during the early phase of allohelper CD4 Tcell priming may result in an enhanced Th2-cell differentiation in vivo. In support of this, we have recently shown that immunization of adult parental strains with semiallogeneic APC, including dendritic cells, in the absence of MHC class I-specific T cells induces a rapid and vigorous expansion of CD4 $\mathrm{T}$ cells secreting type- 2 cytokines and TGF- $\beta$ in response to allogeneic class II MHC products (G. Foucras et al., manuscript submitted for publication). As in other experimental models $(30,31)$, host CD8 T cells could modulate Th2 cell priming by the secretion of IFN- $\gamma$. Indeed, it has

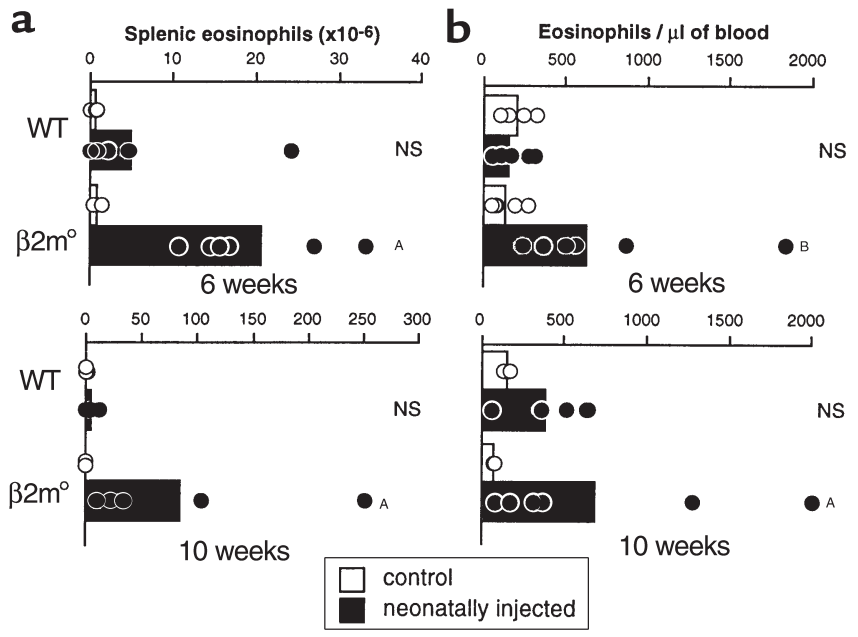

Figure 7

Increased eosinophil number in spleen and blood of $\beta 2 \mathrm{~m}^{\circ} \mathrm{BALB} / \mathrm{c}$ mice injected at birth with $\beta 2 \mathrm{~m}$-deficient CB6F1 splenocytes. Splenic (a) and blood (b) eosinophil numbers were assessed in 6- and 10-week-old control and neonatally injected WT or $\beta 2 \mathrm{~m}^{\circ}$ mice. Bars represent mean values of individual mice (3-9 mice per group). ${ }^{A} P<0.05$; ${ }^{B} P<0.01$.

been shown that the early administration of this cytokine inhibits the Th2-type response induced by the neonatal injection of semiallogeneic spleen cells (32). Finally, there is another subset of MHC class I-restricted T cells, expressing the NK1.1 molecule, that is absent in $\beta 2 \mathrm{~m}^{\circ}$ mice (25). NK1.1 $1^{+} \mathrm{T}$ cells have been reported to ameliorate autoimmune disease (33, 34 ) and acute GVH disease (35) through the production of IL-4. Whether or not this $\mathrm{T}$ cell population play a role in our model is not clear. Lethal HVG disease is associated with uncontrolled Th2 immunity, which would be exacerbated rather than suppressed by IL-4 production from NK T cells. However, as these T-cell subset can also produce IFN- $\gamma$, we cannot exclude that they can effectively control type- 2 Th cells development in $\beta 2 \mathrm{~m}$-sufficient mice.

An important feature of the present study is that the injection of semiallogeneic splenocytes into newborn $\mathrm{BALB} / \mathrm{c}$ mice leads, in the absence of MHC class I-T-cell interactions, to a lethal form of HVG disease. Although, the reasons why mice die during the course of the disease remain to be elucidated, it must be stressed that besides the marked glomerulonephritis, these mice also exhibit sustained blood eosinophilia and an important accumulation of eosinophils not only in their spleen but also in their lungs, making this model relevant to human hypereosinophilia (36). In addition, high numbers of basophils and/or mastocytes were found to be infiltrating the PALS. Eosinophils have been recently shown to mediate rejection of acute and chronic MHC class II-disparate skin allografts in mice $(37,38)$ and are probably responsible for the generation of fibrotic lesions in some chronic inflammatory diseases $(39,40)$. Variable degrees of fibrosis were evident in the spleen of chimeric $\beta 2 \mathrm{~m}^{\circ}$ diseased mice and were associated with eosinophil infiltrates 
indicating that such mechanisms could also be involved in our model. Hypereosinophilia was also associated with an increased capacity of CD4 T cells to secrete IL-5, a cytokine that plays a central role in the differentiation, proliferation, and recruitment of eosinophils (41). Whether or not eosinophils play a major effector role in the observed histological and clinical manifestations, including mortality remains to be elucidated.

In conclusion, we have demonstrated that the HVG reaction that develops in the absence of MHC class I-Tcell interactions leads to a lethal leukoproliferative disorder associated with uncontrolled Th2-type immunity and hypereosinophilic syndrome in mice. These data support the hypothesis that CD8 T cells might play a more active role than previously anticipated by regulating lymphoid chimerism and/or Th2-cell development resulting in a mild and self-limited form of HVG disease. Thus, induction of neonatal lymphoid chimerism in BALB/c mice in the absence of MHC class I-T-cell interactions represents an unique model of Th2-dependent chronic inflammatory disease that may help to understand the mechanisms involved in idiopathic hypereosinophilic syndrome in humans (36).

\section{Acknowledgments}

We thank M. Calise, S. Pilipenko, and M. Marche for their skillful technical assistance. This work was supported by Institut National de la Santé et de la Recherche Médicale (INSERM), and grants from Conseil Général de Région Midi-Pyrénées, Etablissement Français des Greffes, and Université Paul Sabatier. G. Foucras is on a leave of absence from Ecole Nationale Vétérinaire de Toulouse, France. J. Coudert is supported by the Ministère de l'Education Nationale, de la Recherche et de la Technologie. We thank D. Abramowicz for his helpful comments on the manuscript.

1. Billingham, R.E., Brent, L., and Medawar, P.B. 1953. Actively acquired tolerance of foreign cells. Nature. 172:603-606.

2. Feng, H.M., Glasebrook, A.L., Engers, H.D., and Louis, J.A. 1983. Clonal analysis of $\mathrm{T}$ cell unresponsiveness to alloantigens induced by neonatal injection of F1 spleen cells into parental mice. J. Immunol. 131:2165-2169.

3. Nossal, G.J.V., and Pike, B.L. 1981. Functional clonal deletion in immunological tolerance to major histocompatibility complex antigens. Proc. Natl. Acad. Sci. USA. 78:3844-3847.

4. Powell, T.J., and Streilein, J.W. 1990. Neonatal tolerance induction by class II alloantigens activates IL-4-secreting, tolerogen-responsive T cells. J. Immunol. 144:854-859.

5. Schurmans, S., et al. 1990. In vivo effects of anti-IL-4 monoclonal antibody on neonatal induction of tolerance and on associated autoimmune syndrome. J. Immunol. 145:2465-2473.

6. Abramowicz, D., et al. 1990. Persistence of anti-donor allohelper T cells after neonatal induction of allotolerance in mice. Eur. J. Immunol. 20:1647-1653.

7. Chen, N., and Field, E.H. 1995. Enhanced type 2 and diminished type 1 cytokines in neonatal tolerance. Transplantation. 59:933-941.

8. Abramowicz, D., et al. 1990. Increased expression of Ia antigens on B cells after neonatal induction of lymphoid chimerism in mice: role of interleukin 4. Eur. J. Immunol. 20:469-476.

9. Donckier, V., et al. 1995. Critical role of interleukin 4 in the induction of neonatal transplantation tolerance. Transplantation 59:1571-1576.

10. Gao, Q., Chen, N., Rouse, T.M., and Field, E.H. 1996. The role of interleukin-4 in the regulation phase of allogeneic neonatal tolerance. Transplantation. 62:1847-1854.

11. Luzuy, S., Merino, J., Engers, H., Izui, S., and Lambert, P.H. 1986. Autoimmunity after induction of neonatal tolerance to alloantigens: role of B cell chimerism and F1 donor B cell activation. J. Immunol. 136:4420-4426.

12. Goldman, M., et al. 1988. Hyperactivity of donor B cells after neonatal induction of lymphoid chimerism in mice. Clin. Exp. Immunol. 72:79-83.

13. de la Hera, M., et al. 1992. Self-limited autoimmune disease related to transient donor $\mathrm{B}$ cell activation in mice neonatally injected with semi-allogeneic F1 cells. Int. Immunol. 4:67-74.

14. Kühn, R., Rajewsky, K., and Müller, W. 1991. Generation and analysis of interleukin-4 deficient mice. Science. 272:50-54.

15. Takeda, K., et al. 1996. Essential role of Stat6 in IL-4 signalling. Nature. 380:627-630.

16. Shimoda, K., et al. 1996. Lack of IL-4-induced Th2 response and IgE class switching in mice with disrupted Stat6 gene. Nature. 380:630-633.

17. Foucras, G., et al. 1998. $\beta 2$-microglobulin-dependent $T$ cells are not necessary for alloantigen-induced T helper (Th) 2 responses after neonatal induction of lymphoid chimerism in mice. J. Immunol. 161:1751-1757.

18. Koller, B.H., Marrack, P., Kappler, J.W., and Smithies, O. 1990. Normal development of mice deficient in $\beta 2 \mathrm{M}, \mathrm{MHC}$ class I proteins, and $\mathrm{CD}^{+} \mathrm{T}$ cells. Science. 248:1227-1230.

19. Guéry, J.-C., Galbiati, F., Smiroldo, S., and Adorini, L. 1996. Selective development of T helper (Th) 2 cells induced by continuous administration of low dose soluble proteins to normal and $\beta 2$-microglobulin-deficient mice. J. Exp. Med. 183:485-497.

20. Mason, D.W., Pugh, C.W., and Webb, M. 1981. The rat mixed lymphocyte reaction: roles of a dendritic cell in intestinal lymph and T-cell subsets defined by monoclonal antibodies. Immunology. 44:75-87.

21. Tomonari, K., and Spencer, S. 1990. Epitope specific binding of CD8 regulates activation of $\mathrm{T}$ cells and induction of cytotoxicity. Int. Immunol. 2:1189-1194.

22. Reiner, S.L., Zheng, S., Corry, D.B., and Locksley, R.M. 1993. Constructing polycompetitor cDNAs for quantitative PCR. J. Immunol. Methods. 165:37-46.

23. Roehm, N.W., et al. 1984. Interleukin-induced increase in Ia expression by normal mouse B cells. J. Exp. Med. 160:679-694.

24. Noelle, R., Krammer, P.H., Ohara, J., Uhr, J.W., and Vitetta, E.S. 1984 Increased expression of Ia antigens on resting B cells: an additional role for B-cell growth factor. Proc. Natl. Acad. Sci. USA. 81:6149-6153.

25. Bendelac, A., Rivera, M.N., Park, S.-H., and Roark, J.H. 1997. Mouse CD1specific NK1 T cells: development, specificity, and function. Annu. Rev. Immunol. 15:535-562.

26. Apasov, S., and Sitkovsky, M. 1993. Highly lytic CD8+, alpha beta T-cell receptor cytotoxic $\mathrm{T}$ cells with major histocompatibility complex (MHC) class I antigen-directed cytotoxicity in beta 2-microglobulin, MHC class Ideficient mice. Proc. Natl. Acad. Sci. USA. 90:2837-2841.

27. Glas, R., Öhlén, C., Höglund, P., and Kärre, K. 1994. The CD8 ${ }^{+}$T cell repertoire in $\beta 2$-microglobulin-deficient mice is biased toward reactivity against self-major histocompatibility class I. J. Exp. Med. 179:661-672.

28. Le Gros, G., and Erard, F. 1994. Non-cytotoxic, IL-4, IL-5, IL-10 producing CD8+ T cells: their activation and effector functions. Curr. Opin. Immunol. 6:453-457.

29. Merino, J., et al. 1987. Autoimmune syndrome after induction of neonatal tolerance to alloantigens: effects of in vivo treatment with anti-T cell subset monoclonal antibodies. J. Immunol. 139:1426-1431.

30. Srikiatkhachorn, A., and Braciale, T.J. 1997. Virus-specific CD8 ${ }^{+} \mathrm{T}$ lymphocytes downregulate $T$ helper cell type 2 cytokine secretion and pulmonary eosinophilia during experimental murine respiratory syncytial virus infection. J. Exp. Med. 186:421-432.

31. Hussell, T., Baldwin, C.J., O'Garra, A., and Openshaw, P.J.M. 1997. CD8+ T cells control Th2-driven pathology during pulmonary respiratory syncytial virus infection. Eur. J. Immunol. 27:3341-3349.

32. Donckier, V., et al. 1994. IFN- $\gamma$ prevents Th2 cell-mediated pathology after neonatal injection of semiallogenic spleen cells in mice. J. Immunol 153:2361-2368.

33. Gombert, J.M., et al. 1996. Early quantitative and functional deficiency of $\mathrm{NK}^{+}$-like thymocytes in the NOD mouse. Eur. J. Immunol. 26:2989-2998

34. Hammond, K.J.L., et al. 1998. $\alpha / \beta$-T cell receptor (TCR) ${ }^{+}$CD4-CD8 $^{-}$(NKT) thymocytes prevent insulin-dependent diabetes mellitus in nonobese diabetic (NOD)/Lt mice by the influence of interleukin (IL)-4 and/or IL-10.J. Exp. Med. 187:1047-1056.

35. Zeng, D., et al. 1999. Bone marrow NK1.1 ${ }^{-}$and NK1.1 ${ }^{+} \mathrm{T}$ cells reciprocally regulate acute graft versus host disease. J. Exp. Med. 189:1073-1081.

36. Weller, P.F., and Bubley, G.J. 1994. The idiopathic hypereosinophilic syndrome. Blood. 83:2759-2779.

37. Le Moine, A., et al. 1999. Critical roles for IL-4, IL-5, and eosinophils in chronic skin allograft rejection. J. Clin. Invest. 103:1659-1667.

38. Le Moine, A., et al. 1999. IL-5 mediates eosinophilic rejection of major histocompatibility complex class II-disparate skin allografts in mice. $J$. Immunol. 163:3778-3784.

39. Kroegel, C., Virchow, J.-C., Luttmann, W., Walker, C., and Warner, J.A. 1994. Pulmonary immune cells in health and disease: the eosinophil leucocyte (Part I). Eur. Respir. J. 7:519-543.

40. Rothenberg, M.E. 1998. Eosinophilia. N. Engl.J. Med. 338:1592-1600.

41. Sanderson, C.J. 1992. Interleukin-5, eosinophils, and disease. Blood. 79:3101-3109. 